\title{
Addition of computed tomography chest increases the diagnosis rate in patients with suspected intestinal tuberculosis
}

\author{
Saurabh Kedia ${ }^{1}$, Raju Sharma², Sudheer Kumar Vuyyuru ${ }^{1}$, Deepak Madhu ${ }^{1}$, Pabitra Sahu ${ }^{1}$, Bhaskar Kante ${ }^{1}$, \\ Prasenjit Das ${ }^{3}$, Ankur Goyal ${ }^{3}$, Karan Madan $^{4}$, Govind Makharia ${ }^{1}$, Vineet Ahuja ${ }^{1}$ \\ Departments of ${ }^{1}$ Gastroenterology and Human Nutrition, ${ }^{2}$ Radiodiagnosis, ${ }^{3}$ Pathology, and ${ }^{4}$ Pulmonary Medicine, All India Institute of Medical \\ Sciences, New Delhi, India
}

Background/Aims: Intestinal tuberculosis (ITB) is difficult to diagnose due to poor sensitivity of definitive diagnostic tests. ITB may be associated with concomitant pulmonary tuberculosis (PTB) which may remain undetected on chest X-ray. We assessed the role of contrast enhanced computed tomography (CECT) chest in detecting the prevalence of active PTB, and increasing the diagnostic yield in patients with suspected ITB. Methods: Consecutive treatment naïve patients with suspected ITB $(n=200)$ who underwent CECT chest $(n=88)$ and had follow-up duration $>1$ year were recruited in this retrospective study (February 2016 to October 2018). ITB was diagnosed in the presence of caseating granuloma, positive acid fast stain or culture for Mycobacterium tuberculosis on biopsy, presence of necrotic lymph nodes (LNs) on CT enterography or positive response to anti-tubercular therapy. Evidence of active tuberculosis on CECT-chest was defined as presence of centrilobular nodules with or without consolidation/miliary nodules/thick-walled cavity/enlarged necrotic mediastinal LNs. Results: Sixty-five of eightyeight patients (mean age, $33.8 \pm 12.8$ years; $47.7 \%$ of females) were finally diagnosed as ITB (4-caseating granuloma on biopsy, 12-necrotic LNs on CT enterography, 1-both, and 48-response to anti-tubercular therapy) and 23 were diagnosed as Crohn's disease. Findings of active TB on CECT chest with or without necrotic abdominal LNs were demonstrated in 5 and 20 patients, respectively. No patient with Crohn's disease had necrotic abdominal LNs or active PTB. Addition of CECT chest in the diagnostic algorithm improved the sensitivity of ITB diagnosis from $26.2 \%$ to $56.9 \%$. Conclusions: Addition of CECT chest significantly improves the sensitivity for definite diagnosis in a patient with suspected ITB. (Intest Res 2022;20:184-191)

Key Words: Intestinal tuberculosis; Crohn disease; Computed tomography; Diagnosis

\section{INTRODUCTION}

Ever since the diagnostic criteria for intestinal tuberculosis (ITB) were laid down by Logan in 1964, ${ }^{1}$ there have been minimal reforms in these criteria, and in lieu of the poor sensitivity of these criteria, the diagnosis of ITB remains a challenge. This problem is further compounded by the increasing disease

Received August 19, 2020. Revised January 17, 2021.

Accepted January 22, 2021.

Correspondence to Vineet Ahuja, Department of Gastroenterology and

Human Nutrition, All India Institute of Medical Sciences, Ansari Nagar, New

Delhi 110029, India. Tel: +91-11-26594615, Fax: +91-11-26588663, E-mail:

vineet.aiims@gmail.com burden of Crohn's disease (CD), a close mimicker of ITB. ${ }^{2}$ Because of poor sensitivity of diagnostic techniques for ITB and lack of specificity for diagnostic criteria for $\mathrm{CD}$, differentiating $\mathrm{CD}$ and ITB remains challenging, and this dilemma is often resolved by a therapeutic anti-tubercular therapy (ATT) trial. $^{3}$ However, this therapeutic ATT trial is associated with the risk of ATT induced adverse events, notably hepatotoxicity, delay in the diagnosis of $\mathrm{CD}$, and as recently demonstrated by us, complicated course of patients eventually diagnosed with $\mathrm{CD}{ }^{4}$ Hence there is a constant need for improvement in diagnostic strategies for ITB.

Up to one-fourth of patients with ITB have evidence of old/ 
active TB on simultaneous chest X-ray. ${ }^{5}$ Contrast enhanced computed tomography (CECT) chest has better sensitivity than chest X-ray to detect parenchymal as well/mediastinal lesions suggestive of active TB. ${ }^{6-8}$ CECT chest, if done in patients with suspected ITB, would help in classifying the disease accurately at the outset itself as ITB. This would also increase the confidence of clinicians in starting ATT in a patient with suspected ITB.

The present study was therefore aimed to evaluate the role of CECT chest during the initial workup of ITB.

\section{METHODS}

\section{Patients}

Consecutive patients with suspected ITB followed up at the Department of Gastroenterology at the All India Institute of Medical Sciences, New Delhi, India from February 2016 to October 2018 were screened for inclusion in the study. The patients were included if they underwent CECT chest prior to starting ATT, remained on regular follow-up (at least 1 year after ATT) and had repeat evaluation for endoscopic/radiologic healing after completion of ATT. Permission from the Institute Ethics Committee of All India Institute of Medical Sciences was obtained (IEC-52/04.01.2019) and the informed consent was waived.

\section{Study Design}

This retrospective analysis of a prospectively maintained database screened 200 consecutive patients with suspected diagnosis of ITB of which 88 underwent CECT of chest before ATT was started and had a follow-up of 1 year after ATT. At our center, all patients with suspected ITB are subjected to a uniform evaluation including baseline complete blood count, liver function tests, renal function tests, chest X-ray, cross-sectional imaging (computed tomography/magnetic resonance [CT/MR] enterography), endoscopy (upper gastrointestinal endoscopy/ileocolonoscopy according to suspected site of involvement) and biopsy for histopathological examination, acid fast bacillus (AFB), culture and polymerase chain reaction for $M y$ cobacterium tuberculosis. CT/MR enterography is done according to a predefined protocol followed at our center. In addition, a CECT chest was also done for these patients. The data was compiled on a predefined proforma on demographics, mode of diagnosis of ITB, disease duration, clinical, endoscopic, histologic and radiologic features, clinical and radiologic/ endoscopic response to ATT.

\section{Diagnosis of ITB}

The diagnosis of ITB was made in an appropriate clinical setting with the demonstration of necrotizing granulomas on histopathology, demonstration of AFB on histopathology or culture of intestinal tissue, and presence of necrotic lymph nodes on CT enterography (CTE) ${ }^{1,9}$ In patients who did not fulfill the above definitions, therapeutic trial with ATT was given. Diagnosis of ITB was made if patient had clinical and endoscopic/ radiologic response to ATT after completion of ATT at 6 to 9 months. These defining criteria for diagnosis of ITB have been validated earlier in another study at our center. In patients not responding to ATT, response to CD specific therapy confirmed the diagnosis of $\mathrm{CD}{ }^{3}$

\section{Treatment and Follow-up}

Induction regimen of isoniazid $5 \mathrm{mg} / \mathrm{kg}$, rifampicin $10 \mathrm{mg} / \mathrm{kg}$, pyrazinamide $20-25 \mathrm{mg} / \mathrm{kg}$ and ethambutol $15-20 \mathrm{mg} / \mathrm{kg}$ for 2 months followed by maintenance therapy of isoniazid and rifampicin for duration of 4-7 months was given. The total treatment duration was 6-9 months depending upon the response to ATT. Patients were routinely followed up at 2 monthly intervals and as and when required to assess symptom response and also to monitor drug toxicity by assessment of liver function tests. Repeat endoscopy/cross-sectional imaging were done to assess mucosal healing (on endoscopy) or radiologic resolution.

\section{Definitions}

(1) Ulceroconstrictive disease: gastrointestinal disease which occurs due to ulcers or stricture in the small or large intestine; (2) Suspected ITB: patients with ulceroconstrictive intestinal disease with clinical/endoscopic/radiologic features suggestive of ITB; (3) Definite ITB: patients with suspected ITB who fulfilled the diagnostic criteria for ITB; (4) Indeterminate ITB: patients with suspected ITB who did not fulfill the diagnostic criteria for ITB; (5) Mucosal healing: mucosal healing was defined as absence of any evidence of ulceration, erythema (suggestive of active inflammation) on follow-up colonoscopy. Radiologically absence of any signs of inflammatory stricture was also classified as mucosal healing; or (6) Active tuberculosis on CT chest: active tuberculosis on CT chest was defined in the presence of air-space nodules/centrilobular nodules/ clustered nodules or consolidation in apical and posterior segments of right upper lobe, apicoposterior segment of left upper lobe/right middle lobe/lingula, and superior segment in any lower lobe, miliary nodules, thick-walled cavity, cavity 
with surrounding consolidation, enlarged mediastinal lymph nodes with central necrosis (rim enhancement) or heterogeneous enhancement. ${ }^{8}$

\section{Statistical Analysis}

Continuous variables were expressed as median with interquartile range $(\mathrm{IQR})$ or mean \pm standard deviation, depending upon normal or non-normal distribution, and categorical variables were expressed as percentages. McNemar test of proportions was used to compare the sensitivity of definite ITB diagnosis with and without CT chest. SPSS software version 20.0 (IBM Corp., Armonk, NY, USA) and STATA software version 12 (StataCorp, College Station, TX, USA) was used for analysis.

\section{RESULTS}

A total of 200 treatment naïve patients with suspected ITB were evaluated between February 2016 till October 2018. Of these, 88 patients underwent CECT chest before ATT and had a follow-up of 1 year after ATT. Of these 88 patients, 65 were diagnosed with ITB and 23 were diagnosed with CD.

\section{Baseline Demographic, Clinical, Endoscopic and Radiologic Characteristics}

The mean age and median disease duration of patients with ITB were $33.8 \pm 12.8$ years and 12 months (IQR, $6-24$ months) respectively. Thirty-four patients (52.3\%) were males. The most

Table 1. Clinical and Demographic Features of Patients with Intestinal Tuberculosis

\begin{tabular}{lc}
\hline Variable & Intestinal tuberculosis $(\mathrm{n=65})$ \\
\hline Age $(\mathrm{yr})$ & $33.8 \pm 12.8$ \\
Female sex & $31(47.7)$ \\
Duration of symptoms (mo) & $12(6-24)$ \\
Fever & $20(30.8)$ \\
Diarrhea & $14(21.5)$ \\
Constipation & $22(33.8)$ \\
Gastrointestinal bleeding & $9(13.8)$ \\
Pain abdomen & $60(92.3)$ \\
Partial bowel obstruction & $27(41.5)$ \\
Weight loss & $41(63.1)$ \\
Anemia & $45(69.2)$ \\
\hline
\end{tabular}

Values are presented as mean \pm standard deviation, number (\%), or median (interquartile range). common symptom was abdominal pain, seen in $92.3 \%$ of patients followed by weight loss (63.1\%) and recurrent partial bowel obstruction (41.5\%). Fever was present in $30.8 \%$ of patients (Table 1). On colonoscopy, caecum and terminal ileum was the most commonly involved site, seen in $40.0 \%$ and $51.2 \%$ patients respectively, and presence of transverse ulcer was the most common endoscopic feature, seen in $65.5 \%$ patients (Table 2). On histology, granulomas were seen in $39.6 \%$ patients, while caseation was seen in only $8.6 \%$ patients. The median granuloma size was $300 \mu \mathrm{m}$ (IQR, 130-400 $\mu \mathrm{m}$ ). Microgranuloma $(<200 \mu \mathrm{m})$ was present in 5 patients. On CTE, $43.1 \%$ of patients had evidence of stricture, $61.5 \%$ of patients had short segment involvement, mural stratification was seen in $36.9 \%$ of patients and evidence of necrotic lymph nodes was seen in $20 \%$ of patients ( $n=13$ ) (Fig. 1). Twenty-five patients had evidence of active tuberculosis on CT chest (Fig. 2), of which 18 had parenchymal lesions and 3 had evidence of necrotic lymph nodes and 4 had both findings (Table 3 ). Nineteen patients with ITB $(29 \%)$ and 5 patients with CD $(22 \%)$ had evidence of prior TB in the form of fibrotic lesions and calcified granulomas on CT chest.

Table 2. Endoscopic Features of Patients with Intestinal Tuberculosis

\begin{tabular}{lc}
\hline Variable & Intestinal tuberculosis $(n=65)$ \\
\hline Site of involvement & $3 / 58(5.2)$ \\
Rectum & $3 / 58(5.2)$ \\
Sigmoid colon & $3 / 58(5.2)$ \\
Descending colon & $9 / 58(15.5)$ \\
Transverse colon & $14 / 57(24.6)$ \\
Ascending colon & $20 / 50(40.0)$ \\
Caecum & $22 / 43(51.2)$ \\
Terminal ileum & $18 / 50(36.0)$ \\
lleocecal valve & \\
Type of involvement & $12 / 57(21.1)$ \\
Aphthous ulcers & $3 / 55(5.5)$ \\
Longitudinal ulcers & $36 / 55(65.5)$ \\
Transverse ulcers & $3 / 58(5.2)$ \\
Pseudopolyps & $6 / 58(10.3)$ \\
Nodularity & $19 / 58(32.7)$ \\
Stricture & \\
Histology & $23 / 58(39.6)$ \\
Granuloma & $5 / 58(8.6)$ \\
Caseation &
\end{tabular}

Values are presented as number/number (\%). 


\section{Diagnostic Criteria for TB}

Of 88 patients, 65 were diagnosed with ITB, of which 4 patients had evidence of caseating granulomas on biopsy, and 12 had presence of necrotic lymph nodes on CTE, and 1 had both, confirming the diagnosis of ITB. In rest of the patients, the diagnosis was confirmed on follow-up, with documentation of clinical and endoscopic/radiologic response. Therefore, 73.8\% patients required therapeutic ATT trial. Of 13 patients with necrotic lymph nodes on CTE, imaging was repeated in 6 , and 5 out of 6 patients showed resolution of these necrotic lymph nodes.

Twenty-three patients were eventually diagnosed as CD, because of non-response to ATT and improvement with CD specific therapy.

\section{Role of CECT Chest in the Diagnosis of ITB}

Of 65 patients with ITB, 20 patients had evidence of active tuberculosis on CT chest, and 5 had evidence of both necrotic
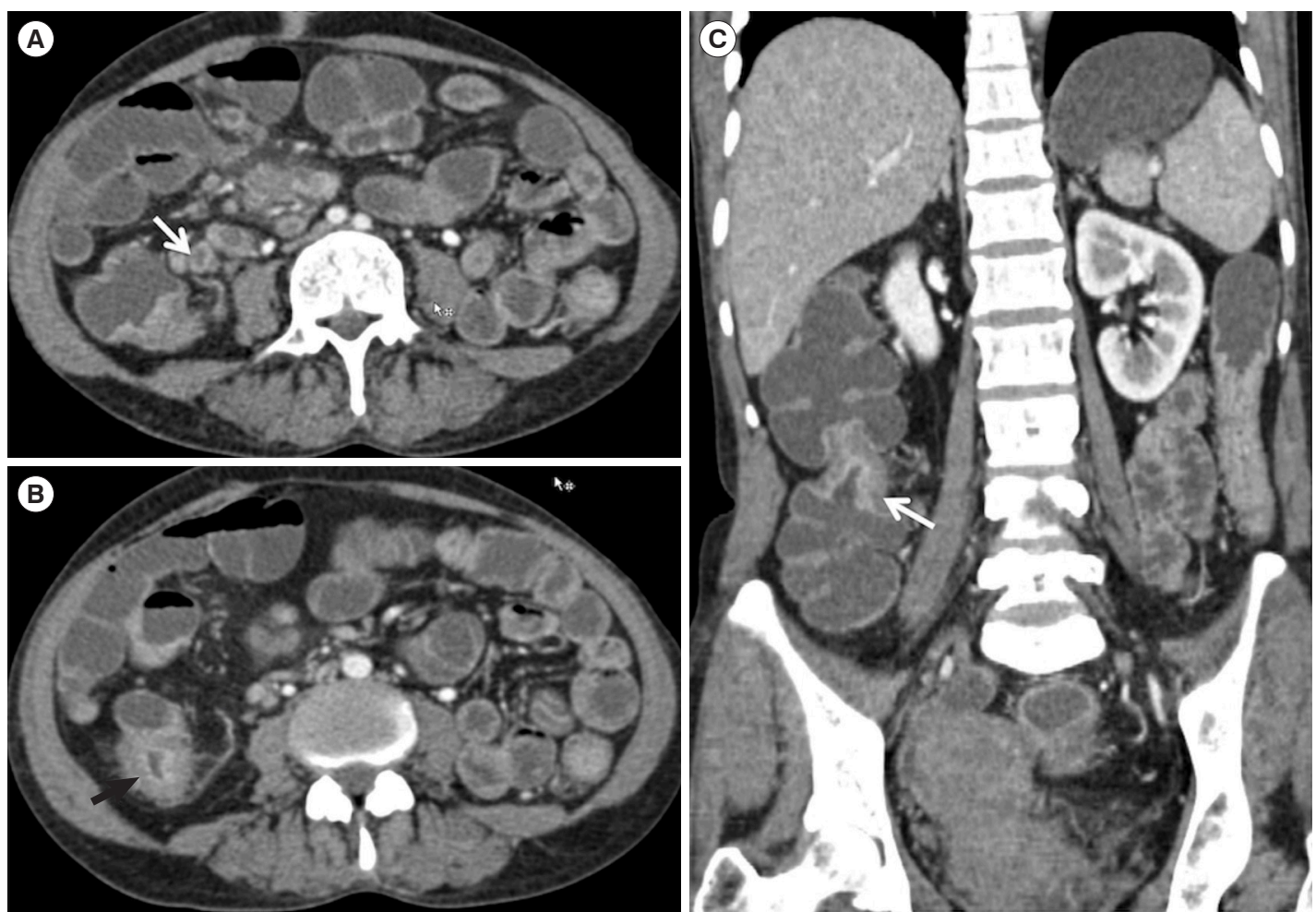

Fig. 1. (A, B) Axial computed tomography (CT) enterography images showing enlarged lymph nodes with central necrosis and peripheral enhancement in the pericolic region (white arrow) along with colonic thickening (black arrow). (C) Coronal reformatted CT enterography image showing inflammatory stricture in the ascending colon (white arrow).
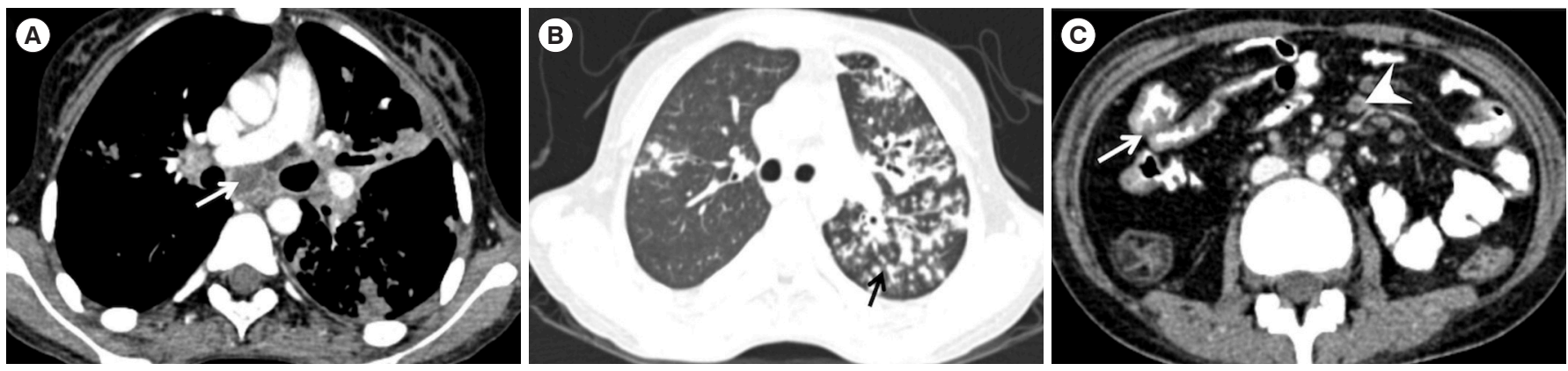

Fig. 2. Axial contrast enhanced computed tomography (CECT) mediastinal window (A) and lung window (B) showing necrotic subcarinal lymph node (white arrow) and acinar nodules some of which show tree-in-bud morphology (black arrow). (C) Axial CECT abdomen with positive oral contrast showing thickening of ileal loops (white arrow) and few necrotic mesenteric lymph nodes (white arrowhead). 
Table 3. CT Findings in Patients with ITB

\begin{tabular}{|c|c|}
\hline Variable & ITB $(n=65)$ \\
\hline \multicolumn{2}{|l|}{ Site of involvement } \\
\hline Left colon ${ }^{a}$ & $1(1.5)$ \\
\hline Colon $^{b}$ & $8(12.3)$ \\
\hline Ileocecal area ${ }^{c}$ & $32(49.2)$ \\
\hline Rest of small intestine ${ }^{d}$ & $20(30.8)$ \\
\hline \multicolumn{2}{|l|}{ Type of involvement } \\
\hline Long segment involvement & $25(38.5)$ \\
\hline Asymmetric thickening & $8(12.3)$ \\
\hline Mural stratification & $24(36.9)$ \\
\hline Stricture & $28(43.1)$ \\
\hline Mesenteric changes & $29(44.6)$ \\
\hline Comb sign & 13 \\
\hline Fat proliferation & 16 \\
\hline Necrotic abdominal lymph nodes & $13(20.0)$ \\
\hline Active TB on CT chest & $25(38.5)$ \\
\hline Parenchymal lesions & 18 \\
\hline Necrotic mediastinal lymph nodes & 3 \\
\hline Both & 4 \\
\hline $\begin{array}{l}\text { Active TB on CT chest in patients with } \\
\text { definite TB }(n=17)\end{array}$ & $7(41.2)$ \\
\hline $\begin{array}{l}\text { Active TB on CT chest in patients without } \\
\text { definite TB }(n=48)\end{array}$ & $18(37.5)$ \\
\hline$P$-value & 0.79 \\
\hline
\end{tabular}

Values are presented as number (\%) or number.

a Left colon includes descending colon, sigmoid colon, rectum.

${ }^{b}$ Colon includes caecum (without contiguous terminal ileal involvement), ascending colon, transverse colon and left colon.

'Rest of small intestine includes jejunum, ileum excluding the terminal ileum.

dlleocecal area includes terminal ileum just adjacent to the ileocecal valve or contiguous terminal ileal and cecal involvement.

TB, tuberculosis; ITB, intestinal tuberculosis; $C T$, computed tomography.

lymph nodes on CTE and active TB on CECT chest, while none of the 23 patients with CD either had either evidence of active TB on CT chest or necrotic lymph nodes on CTE. Of 25 patients with positive CECT chest, only 1 was symptomatic for pulmonary TB. Bronchoalveolar lavage for AFB was done in 3 patients, and was positive in 1 patient. Repeat imaging done in 3 patients demonstrated complete healing of chest lesions with ATT.

Therefore, if CECT chest findings were included in the diagnostic criterion for ITB, then in the present study, patients who would be diagnosed with ITB at the onset would increase from
17 of 65 to 37 of 65 , increasing the diagnostic sensitivity of available investigations (including CT findings) for ITB from 26.1\% to $56.9 \%$, which was statistically significant $(P<0.001)$.

\section{DISCUSSION}

Abdominal tuberculosis is the sixth most common form of extrapulmonary tuberculosis (EPTB), ${ }^{10}$ and ITB is the major manifestation of abdominal tuberculosis. Except for lymph node tuberculosis, diagnosing EPTB has always remained a challenge, primarily because of its paucibacillary nature, which makes it difficult to isolate the organism, the demonstration of which is a definite diagnostic criterion for any infectious disease. Caseation necrosis on biopsy, another pathognomonic hallmark of EPTB is again a rare entity, ${ }^{11}$ and therefore the sensitivity for diagnosis of EPTB/ITB has always remained low. Further, if the involvement in ITB is limited to small bowel only, then obtaining biopsy samples becomes difficult and these definitive microbiological/pathological tests cannot be applied. Presence of necrotic lymph nodes on CTE adds to the diagnostic accuracy, but this feature is also limited by poor sensitivity, as shown in a recent meta-analysis. ${ }^{12}$ This diagnostic gap for EPTB has been circumvented by the so called composite reference standard which includes demonstration of clinical response to ATT after the therapeutic ATT trial. However, because of diagnostic confusion with CD and presence of ATT response in a proportion of patients with $\mathrm{CD},{ }^{3}$ the composite reference standard for ITB also includes the demonstration of endoscopic/radiologic response to ATT. However, the therapeutic trial with ATT is associated with the short-term risk of ATT induced hepatotoxicity, and as recently reported by us, long term risk of complicated disease course in patients eventually diagnosed with $\mathrm{CD} .{ }^{4}$ Hence, there is a need for better diagnostic modalities for upfront definite diagnosis of ITB. ${ }^{13-15}$ One of these could be the demonstration of active tuberculosis at extraintestinal site, and the most common site would be pulmonary tuberculosis, as shown in the present study, where addition of CECT chest improved the sensitivity for diagnosing ITB.

Of 88 patients with suspected ITB, 65 were finally diagnosed with ITB, and in $73.8 \%$ of patients, the basis for diagnosis was therapeutic response to ATT. Of the definite diagnostic tests for ITB, caseating granulomas were positive in 5 patients, and necrotic lymph nodes on CT were present in 13 patients. These necrotic lymph nodes were not subjected to tissue diagnosis because the underlying clinical setting was strong for ITB, and 
5 out of 6 patients in whom imaging was repeated, showed resolution of necrotic lymph nodes. Thus, overall sensitivity of ITB diagnosis was only $26.2 \%$, which was is comparable to other series.

The most interesting finding of this study was the demonstration of active tuberculosis on CECT chest in $38.5 \%$ of patients with ITB, with only 1 patient being symptomatic for pulmonary TB. Recent guidelines on EPTB have suggested a chest $\mathrm{X}$-ray in the diagnostic algorithm of a patient with suspected EPTB, ${ }^{16}$ and multiple series on ITB have demonstrated evidence of old/active TB on chest X-ray in up to $25 \%$ patients. ${ }^{5}$ Further, multiple series on CD/ITB differentiation have suggested demonstration of active pulmonary $\mathrm{TB}$ in patients with ITB as the definite differentiating feature between the 2 conditions. ${ }^{17,18}$ The present study has extended this observation by utilizing a more sensitive and a definitive diagnostic technique for detecting associated pulmonary lesions in patients with ITB.

Inclusion of positive CT chest findings as a definite diagnostic criterion significantly increased the sensitivity of diagnosing ITB, from $26 \%$ to $57 \%$, which suggests for the inclusion of CT chest in the diagnostic algorithm of a patient with suspected ITB (Fig. 3). However, there is a risk of radiation with additional CTs, and we therefore suggest CT chest in patients where the definitive diagnostic criteria including necrotic lymph nodes are not met and the chest X-ray is inconclusive (Fig. 3). Presence of active TB on CT chest would increase the confidence in diagnosis of ITB and initiation of ATT. Patients who are initiated on ATT in the absence of these criteria (therapeutic ATT trial) need to be followed up vigilantly with early assessment for endoscopic/clinical response, as we have recently shown that ATT in patients with eventual diagnosis of CD predisposes to stricture formation. ${ }^{4}$

The study is limited by its retrospective design and small sample size. However, the data was collected from a prospectively maintained database and included consecutive patients with suspected ITB who underwent CECT chest before ATT was administered and had a follow-up of more than 1 year. One year of follow-up ensured that the diagnosis of ITB was definite in those patients who were administered therapeutic ATT trial and sustained their response till 1 year. There was lack of histological/microbiological proof of TB either from necrotic lymph nodes or from CT chest. However, search for definite TB in this setting would involve invasive techniques, increase the time to diagnosis and because of their poor sensitivity, absence of definite evidence will not refute the diagnosis

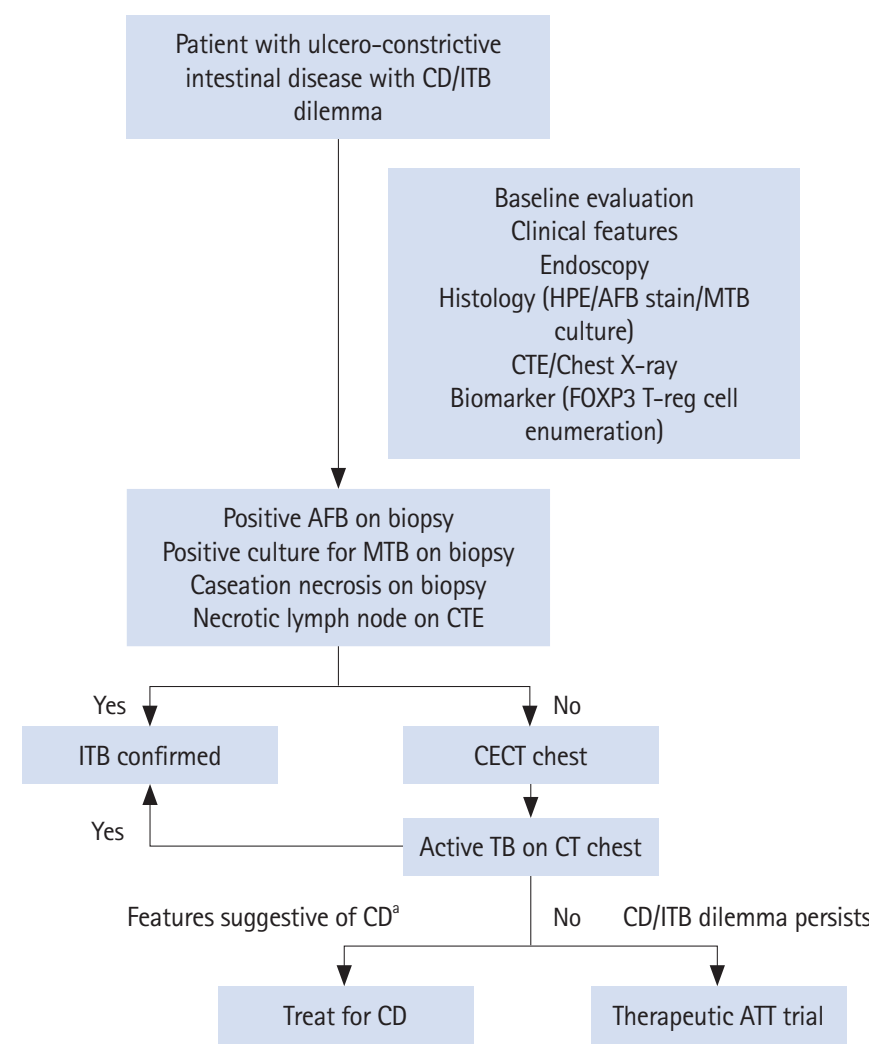

Fig. 3. Diagnostic algorithm in a patient with suspected ITB incorporating CT chest. ${ }^{a}$ Features suggestive CD: (1) Clinical: diarrhea/perianal lesions/bleeding per-rectum; (2) Endoscopy: linear ulcers/cobblestoning; (3) CTE: skip lesions/comb sign/long segment involvement/increased visceral fat; or (4) Biomarker: low peripheral blood FOXP3 T regulatory cells. CD, Crohn's disease; ITB, intestinal tuberculosis; HPE, histopathology; AFB, acid fast bacillus; MTB, Mycobacterium tuberculosis; CTE, computed tomography enterography; CECT, contrast enhanced computed tomography; $\mathrm{TB}$, tuberculosis; $\mathrm{AT}$, anti-tubercular therapy.

of ITB. Moreover, the diagnosis of active TB on CT chest was entertained after consulting pulmonologist and we could demonstrate radiological healing after ATT in a fraction of patients. The results also require validation in a large sample size from other centers.

Though, including only patients with suspected ITB could have biasedly increased the chance of positive findings on CT chest, the primary objective of this study was to evaluate the role of CT chest in increasing upfront diagnosis rate in patients with suspected ITB. Hence, the study provides a simple message that among patients with suspected ITB who do not fulfill the definitive diagnostic criteria for ITB, addition of CT chest might increase the physician's confidence in the diagnosis of ITB in a particular patient. Moreover, absence of active TB on 
CT chest in patients who were eventually diagnosed with $\mathrm{CD}$, further increases our confidence in the role of CT chest on this aspect.

To conclude, there is a significant improvement in diagnostic sensitivity with the addition of CECT chest findings in the diagnostic algorithm of a patient with suspected ITB.

\section{ADDITIONAL INFORMATION}

\section{Funding Source}

This study was supported by the Indian Council of Medical Research: Centre for advanced research and excellence in intestinal diseases (grant number: 55/4/11/CARE-ID/2018-NCDII).

\section{Conflict of Interest}

No potential conflict of interest relevant to this article was reported.

\section{Author Contribution}

Conceptualization: Ahuja V. Data curation: Kedia S, Vuyyuru SK, Madhu D. Formal analysis: Kedia S, Vuyyuru SK, Goyal A. Investigation: Sharma R. Methodology: Goyal A, Ahuja V. Project administration, supervision: Ahuja V. Writing - original draft: Kedia S, Makharia G, Ahuja V. Writing - review \& editing: Sharma R, Vuyyuru SK, Madhu D, Sahu P, Kante B, Das P, Goyal A, Madan K, Makharia G, Ahuja V. Approval of final manuscript: all authors.

\section{ORCID}

Kedia S

Sharma R

Vuyyuru SK

Madhu D

Sahu P

Kante B

Das P

Goyal A

Madan K

Makharia G

Ahuja V

https://orcid.org/0000-0002-5758-0144 https://orcid.org/0000-0001-5181-263X https://orcid.org/0000-0002-8281-3237 https://orcid.org/0000-0002-8613-8670 https://orcid.org/0000-0002-9847-0136 https://orcid.org/0000-0001-5363-7273 https://orcid.org/0000-0002-2420-8573 https://orcid.org/0000-0003-3333-3701 https://orcid.org/0000-0002-5330-6391 https://orcid.org/0000-0002-2474-2194 https://orcid.org/0000-0002-1577-0118

\section{REFERENCES}

1. Logan VS. Anorectal tuberculosis. Proc R Soc Med 1969;62: 1227-1230.
2. Singh P, Ananthakrishnan A, Ahuja V. Pivot to Asia: inflammatory bowel disease burden. Intest Res 2017;15:138-141.

3. Pratap Mouli V, Munot K, Ananthakrishnan A, et al. Endoscopic and clinical responses to anti-tubercular therapy can differentiate intestinal tuberculosis from Crohn's disease. Aliment Pharmacol Ther 2017;45:27-36.

4. Gupta A, Pratap Mouli V, Mohta S, et al. Antitubercular therapy given to differentiate Crohn's disease from intestinal tuberculosis predisposes to stricture formation. J Crohns Colitis 2020;14:1611-1618.

5. Sharma MP, Bhatia V. Abdominal tuberculosis. Indian J Med Res 2004;120:305-315.

6. Raniga S, Parikh N, Arora A, Vaghani M, Vora PA, Vaidya V. Is HRCT reliable in determining disease activity in pulmonary tuberculosis? Indian J Radiol Imaging 2006;16:221-228.

7. Alkabab YM, Enani MA, Indarkiri NY, Heysell SK. Performance of computed tomography versus chest radiography in patients with pulmonary tuberculosis with and without diabetes at a tertiary hospital in Riyadh, Saudi Arabia. Infect Drug Resist 2018;11:37-43.

8. Nachiappan AC, Rahbar K, Shi X, et al. Pulmonary tuberculosis: role of radiology in diagnosis and management. Radiographics 2017;37:52-72.

9. Paustian F. Tuberculosis of the intestine. In: Haubrich WS, Schaffner F, Berk JE, eds. Bockus gastroenterology. 5th ed. Philadelphia: Saunders, 1995:3304.

10. Sharma SK, Mohan A. Extrapulmonary tuberculosis. Indian J Med Res 2004;120:316-353.

11. Du J, Ma YY, Xiang H, Li YM. Confluent granulomas and ulcers lined by epithelioid histiocytes: new ideal method for differentiation of ITB and CD? A meta analysis. PLoS One 2014;9: e103303.

12. Kedia S, Sharma R, Sreenivas V, et al. Accuracy of computed tomographic features in differentiating intestinal tuberculosis from Crohn's disease: a systematic review with meta-analysis. Intest Res 2017;15:149-159.

13. Tiwari V, Kedia S, Garg SK, et al. CD4+ CD25+ FOXP3+ T cell frequency in the peripheral blood is a biomarker that distinguishes intestinal tuberculosis from Crohn's disease. PLoS One 2018;13:e0193433.

14. Rampal R, Kedia S, Wari MN, et al. Prospective validation of CD4+CD25+FOXP3+ T-regulatory cells as an immunological marker to differentiate intestinal tuberculosis from Crohn's disease. Intest Res 2021;19:232-238.

15. Yadav DP, Madhusudhan KS, Kedia S, et al. Development and validation of visceral fat quantification as a surrogate marker 
for differentiation of Crohn's disease and intestinal tuberculosis. J Gastroenterol Hepatol 2017;32:420-426.

16. Sharma SK, Ryan H, Khaparde S, et al. Index-TB guidelines: guidelines on extrapulmonary tuberculosis for India. Indian J Med Res 2017;145:448-463.

17. Kedia S, Das P, Madhusudhan KS, et al. Differentiating Crohn's disease from intestinal tuberculosis. World J Gastroenterol 2019;25:418-432.

18. Limsrivilai J, Shreiner AB, Pongpaibul A, et al. Meta-analytic bayesian model for differentiating intestinal tuberculosis from Crohn's disease. Am J Gastroenterol 2017;112:415-427. 